\title{
Slow strain waves in blocky geological media from GPS and seismological observations on the Amurian plate
}

\author{
Victor G. Bykov and Sergey V. Trofimenko \\ Institute of Tectonics and Geophysics, Far Eastern Branch, Russian Academy of Sciences, \\ 65 Kim Yu Chen St., Khabarovsk, 680000, Russia \\ Correspondence to: Victor G. Bykov (bykov@itig.as.khb.ru)
}

Received: 7 September 2016 - Published in Nonlin. Processes Geophys. Discuss.: 27 September 2016

Revised: 23 November 2016 - Accepted: 29 November 2016 - Published: 19 December 2016

\begin{abstract}
Based on the statistical analysis of spatiotemporal distribution of earthquake epicenters and perennial geodetic observation series, new evidence is obtained for the existence of slow strain waves in the Earth. The results of our investigation allow us to identify the dynamics of seismicity along the northern boundary of the Amurian plate as a wave process. Migration of epicenters of weak earthquakes $(2 \leq M \leq 4)$ is initiated by the east-west propagation of a strain wave front at an average velocity of $1000 \mathrm{~km} \mathrm{yr}^{-1}$. We have found a synchronous quasi-periodic variation of seismicity in equally spaced clusters with spatial periods of 3.5 and $7.26^{\circ}$ comparable with the length of slow strain waves. The geodetic observations at GPS sites in proximity to local active faults show that in a number of cases, the GPS site coordinate seasonal variations exhibit a significant phase shift, whereas the time series of these GPS sites differ significantly from a sinusoid. Based on experimental observation data and the developed model of crustal block movement, we have shown that there is one possible interpretation for this fact that the trajectory of GPS station position disturbance is induced by migration of crustal deformation in the form of slow waves.
\end{abstract}

\section{Introduction}

The inhomogeneous blocky structure of the crust and the lithosphere considerably affects the deformation, seismic, filtration and other processes. The effect of the blocky structure on the distribution of earthquakes can be especially clearly traced. It is exactly the blocky structure of the geological medium which results in the generation of waves of different types, including slow strain waves (Bykov, 2008). Clarification of the link between movements of tectonic structures and slow strain wave processes is of fundamental importance for expanding our understanding of the physics of earthquakes.

The most important problem of recent geodynamics is to clarify the mechanisms responsible for the propagation of the energy of deformation processes and tectonic stress transfer at the boundaries between the blocks and the lithospheric plates, and to explore the causes of migration of earthquake epicenters. The problem has been argued for more than 45 years since Elsasser's publication (1969), suggesting the equation of local stress transfer in the rigid elastic lithosphere underlain by the viscous asthenosphere. The possibility of using Elsasser's model to describe migration of seismicity was further discussed in papers published by other researchers. Bott and Dean (1973) introduced the term "stress or strain waves" and obtained the expression for the velocity of the wave propagating along the lithospheric plate. According to their calculation, the stress wave velocity attains 0.1 $100 \mathrm{~km} \mathrm{yr}^{-1}$. Anderson (1975) generalized Elsasser's model in order to elucidate the mechanism of earthquake migration in the subduction zone and estimated the stress wave velocity along the island arc to be about $50-170 \mathrm{~km} \mathrm{yr}^{-1}$. In the model developed by Ida (1974), the solution was obtained in the shape of "slow-moving deformation pulses" propagating along the fault at a constant velocity. The gouge viscosity and thickness variations in the fault yield the pulse velocity ranging from $10-100 \mathrm{~km} \mathrm{yr}^{-1}$ to $1-10 \mathrm{~km} \mathrm{day}^{-1}$. The first interval corresponds to earthquake migration velocities at a wavelength of about tens of kilometers, whereas the second interval is consistent with aseismic creep at about $1 \mathrm{~km}$ wavelength. Scholz (1977) introduced the concept of the "defor- 
mation front" to describe large-scale tectonic processes triggering large earthquakes. As estimated by Scholz, the velocity of the deformation front propagating through northeastern China that triggered the $1975, M=7.3$ Haicheng earthquake attained $110 \mathrm{~km} \mathrm{yr}^{-1}$.

The advances in theoretical studies of slow strain waves in the Earth initiated the search for the possibilities of experimentally detecting the propagation effects of the waves of this type, and, especially, the intense study of earthquake migration. By now, the deformographic, geodetic and hydrological measurements performed worldwide have revealed the migration of deformations at velocities of about 10 $100 \mathrm{~km} \mathrm{yr}^{-1}$ and $1-10 \mathrm{~km} \mathrm{day}^{-1}$ (Kasahara, 1979; Bella et al., 1990; Harada et al., 2003; Kuz'min, 2012; Reuveni et al., 2014; Yoshioka et al., 2015). Migration of earthquake epicenters coincides with the velocity $\left(10-100 \mathrm{~km} \mathrm{yr}^{-1}\right)$ and direction of crustal deformation movement (Kasahara, 1979; Barabanov et al., 1988) and with hydrological effects (Kissin, 2008). Furthermore, the absorption and dispersion of the waveforms of migrating deformation were detected (Kasahara, 1979; Barabanov et al., 1988), i.e., the main properties of a common wave process. In terms of the physical mechanism of propagation, slow strain waves are similar to common seismic waves, but the fundamental difference is that they propagate at super low velocities and ultra low frequencies, and have large wavelengths (Bykov, 2005). This hampers the direct instrumental measurements of strain waves and the concomitant effects.

In the present study, we have obtained new evidence of the existence of strain waves in the Earth resting upon a comprehensive statistical analysis of the dynamics of seismicity along the northern boundary of the Amurian plate and the data derived from in situ GPS experimental observations performed near this boundary.

\section{Methods for detection of slow strain waves}

Slow strain wave transmittance through the fault-blocky geological medium is accompanied by various seismic, hydrogeological, electrokinetic, geochemical and other effects. The methods for strain wave detection are divided into indirect methods that display the wave-shaped variations in the geophysical fields due to temporal variations of the stress state of the medium, and direct ones immediately recording the migration of deformations.

The seismic, geoelectric and geochemical methods of strain wave recording are referred to as the indirect methods. Indirect evidence of the existence of strain waves is manifested in the targeted migration of large earthquakes (Stein et al., 1997), the occurrence of seismic velocity anomalies (Lukk and Nersesov, 1982; Nevskii et al., 1987), a cyclic wandering of aseismic strips in the Earth's mantle (Malamud and Nikolaevsky, 1983, 1985), oscillatory movements of the seismic reflection sites (Bazavluk and Yudakhin, 1993; Bor- motov and Bykov, 1999) and the migration of geophysical field anomalies (radon, electrokinetic signals) in proximity to faults (Nikolaevskiy, 1998).

The direct indications of strain waves are displayed in wave fluctuations of the ground water level and the migration of slopes and surface deformations. The direct methods exploring temporal variations of crustal deformation comprise the deformographic (Kasahara, 1979; Ishii et al., 1983; Nevskii et al., 1987; Bella et al., 1990; Harada et al., 2003), hydrogeodynamic (Barabanov et al., 1988; Kissin, 2008) and geodetic measurements (Kuz'min, 2012), including the methods of deformation measurements using laser ranging (Milyukov et al., 2013) and GPS observations (Reuveni et al., 2014; Yoshioka et al., 2015).

To detect the main physical mechanisms of seismicity migration and the generation of signals of a different nature that accompany strain waves, we need to perform further observations and improve GPS and seismological data processing techniques, and conduct theoretically prepared and purposeful experiments.

The answer to the question "where to search for slow strain waves?" is directly linked with the detection of the main types of tectonic structures generating these waves.

\section{Tectonic structures generating slow strain waves}

From the published results it follows that subduction, collision, active riftogenesis and transform fault zones are the most probable types of tectonic structures generating strain waves. These intensive sources of different tectonic natures possess a common property - they are the interaction zones between crustal blocks and the lithospheric plates.

Migration of shear deformation in subduction zones is directed from the ocean toward the coast. This general tendency was first revealed in the area of the Japan island arc where migration is oriented east-west, and in the opposite Pacific coastal area - in the western Cordilleras, where deformations migrated from south to north (Kasahara, 1979). Migration of the maximum of the vertical crustal deformation from the subduction zone toward the continent at a velocity of about $10 \mathrm{~km} \mathrm{yr}^{-1}$ was also observed near the Tohoku region (northeastern Japan) and the Izu Peninsula (central Japan), where the Pacific and Philippine plates subduct beneath the Eurasian plate (Miura et al., 1989). All these data reasonably lead to an assumption that subduction zones are one of the possible sources of slow strain waves.

The seismicity pattern observed in the south of central Asia can also be explained by strain waves excited under the oscillating regime of the Eurasian and Indian lithospheric plate collision in the Pamir and Tien Shan junction zone (Nersesov et al., 1990). The compression at the Indostan and Eurasian lithospheric plate boundary in the Himalayan collision zone is the source of "fast" and "slow" waves of plastic 


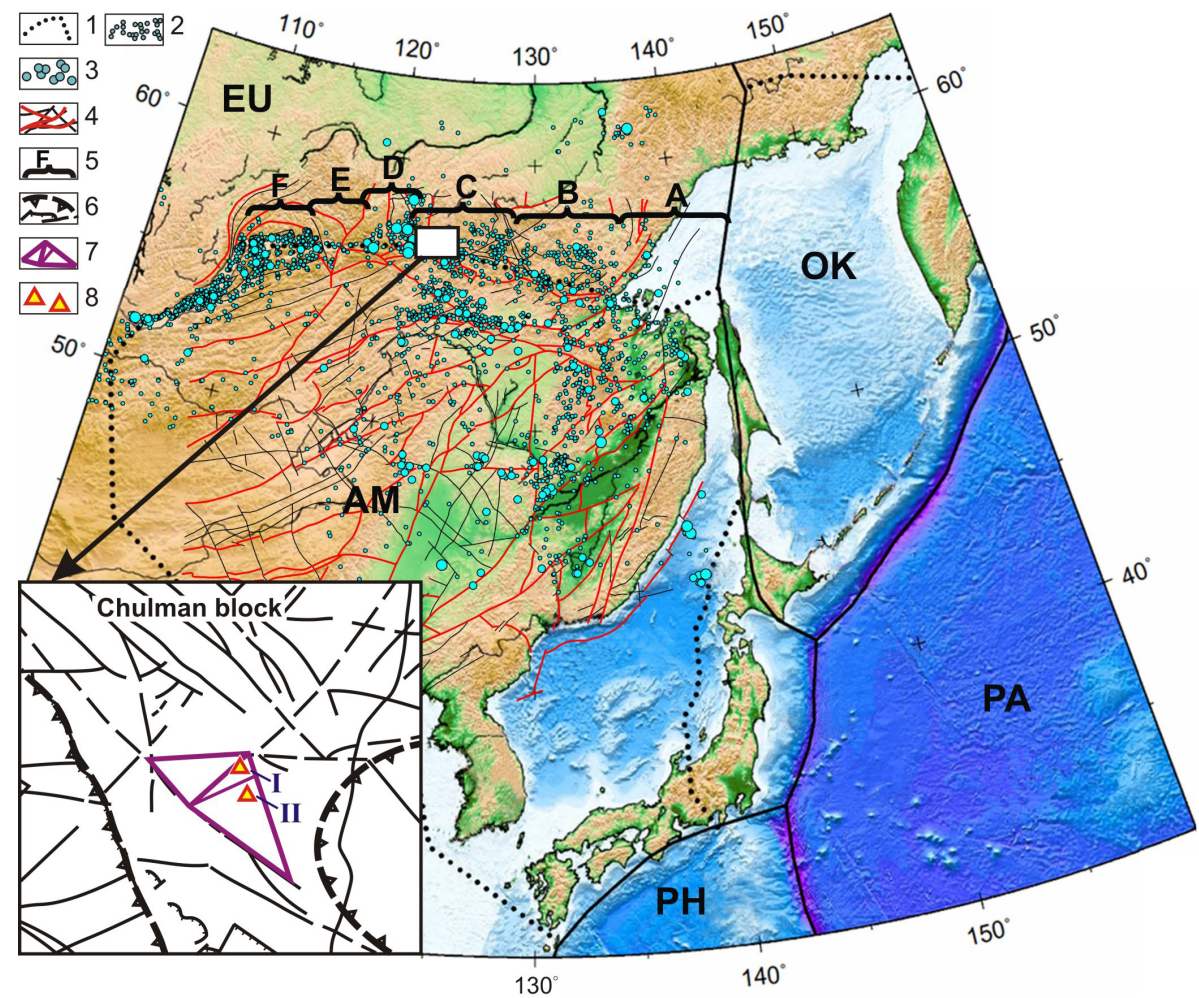

Figure 1. The distribution of earthquake epicenters in the zone of interaction between the Amurian, Eurasian and Okhotsk lithospheric plates; 1 - lithospheric plate boundaries: EU - Eurasian, PA - Pacific, PH - Philippine, OK - Okhotsk; 2 - epicenters of earthquakes with magnitude $M>3 ; 3$ - epicenters of earthquakes with magnitude $M>5 ; 4$ - main tectonic faulting; 5 - spatial cycles of seismicity. A black rectangle shows a sketch map of fault tectonics of the Chulman block, where GPS sites are located: 6 - northeast- and northwest-trending faults of different kinematics; 7 - local block, bordered by active faults; 8 - GPS sites (I - NRGR, II - NRG2).

deformation that trigger earthquakes in central and eastern Asia (Wang and Zhang, 2005).

In the Baikal rift system, four main groups of strain waves with different velocities (7-95 $\mathrm{km} \mathrm{yr}^{-1}$ ) and lengths (130$2000 \mathrm{~km})$ are distinguished that cause recent activation of seismoactive faults in central Asia (Gorbunova and Sherman, 2012).

Based on continuous long-term seismic and laser ranging observation data, the effect of propagation of slow waves of tectonic deformations traveling along transform faults at velocities of $40-50 \mathrm{~km} \mathrm{yr}^{-1}$ at the lithospheric plate boundaries in southern California and the Kopet Dag region has been established (Nevskii et al., 1987). Seismicity variations along the Pacific and North American plate boundary in the San Andreas transform fault zone (California) are also suggested as being associated with "slowly traveling strain waves" (Press and Allen, 1995).

The rotational block movements in the fault zones due to tectonic processes or earthquakes are considered one of the main physical mechanisms of strain wave generation (Nikolaevskiy, 1996; Lee et al., 2009; Teisseyre et al., 2006).

\section{Seismic effects of slow strain waves at the northern edge of the Amurian plate}

In order to specifically investigate the relationship between strain waves and the dynamics and seismicity pattern observed in fault-blocky geological media, we have selected the study area on the northern margin of the Amurian plate - the most seismically active area of the interaction zone between the Amurian and Eurasian plates.

The analysis of the spatiotemporal seismicity pattern observed in vast regions is commonly performed based on statistical processing of earthquake catalogs. The directions of earthquake epicenter (or groups of epicenters) displacements are defined and their displacement rates are determined. As opposed to the standard regional approach, we here applied a comprehensive analysis including both conventional statistical methods and those of cluster analysis adapted by the authors for the geodynamic zone gradation. The details of developed clustering techniques and statistical analysis of background seismicity can be found in Trofimenko et al. (2015). In the paper by Trofimenko et al. (2016a), the statistical validity of the applied method is shown and the correctness of the models is evaluated. 
To study the dynamics of seismicity in different zones, the area along the northern boundary of the Amurian plate was divided into separate clusters (Fig. 1). When clustering, we applied the criterion of earthquake grouping near active faults, and the geomorphological and tectonic features of active structures, as well as the presence of meridional (submeridional) first-rank faults within the distinguished zones, were taken into consideration.

When developing space-time models of seismicity, the spatial relationship between separate seismic clusters during a year was revealed and taken into account. Based on statistical distributions of earthquakes, the analysis of seismicity maxima passage over easterly-westerly arranged clusters has been performed.

The basic data were derived from the Earthquakes of Russia catalog (http://eqru.gsras.ru), the catalog compiled by the Baikal Branch of the Geophysical Survey of the Russian Academy of Sciences (GS RAS) (http://www.seis-bykl.ru/) and the IRIS catalog (http://www.iris.edu).

As a result of the calculation, the average period of seismicity maximum passage in days from the beginning of the year has been determined for each cluster, which is assigned to the average value of the cluster longitude. These values were used for the calculation of the displacement rate of seismicity maxima. We calculated the velocities and wavelengths of slow strain waves from the maxima of the spatial correlation of seismicity.

The spatiotemporal distributions of earthquake epicenters reflect synchronization of seismicity maxima in the annual cycles over a certain spatial interval (migration period). The statistical calculations performed for each cluster allowed the identification of six similar spatiotemporal cycles of seismicity maxima migration A, B, C, D, E and F (Fig. 1), for which the spatial periods of migration and displacement rates of seismicity maxima have been calculated.

In the northeastern segment, the maxima of statistical distributions are located in the clusters arranged nearly equally apart from each other, at $L_{\mathrm{A}-\mathrm{C}}=(7.26 \pm 0.74)^{\circ}$, which corresponds to a distance of $360-420 \mathrm{~km}$ for a range of investigated latitudes. For the northwestern segment, the spatial period is equal to $L_{\mathrm{D}-\mathrm{F}}=(3.8 \pm 0.5)^{\circ}$, on average, which corresponds to half of the interval $L_{\mathrm{A}-\mathrm{C}}$, or a distance of $210-270 \mathrm{~km}$ (Fig. 1). In the study area, the parameter $L_{\mathrm{A}-\mathrm{C}}$ is equal to double the distance between the main structuraltectonic elements of the Earth's crust and corresponds to double the size of tectonic inhomogeneities revealed from the geophysical field anomalies (Trofimenko, 2010).

The determined spatial period $L_{\mathrm{A}-\mathrm{C}}=7.26^{\circ} \quad(360-$ $420 \mathrm{~km}$ ) is comparable with the wavelength $\lambda=250-450 \mathrm{~km}$ of slow strain waves observed in the study area on the northern margin of the Amurian plate (Pribaikalya and Priamurye areas lying within $107-140^{\circ} \mathrm{E}$ ) (Sherman et al., 2011). The direction of the seismicity maxima displacement coincides with the displacement vector of the strain wave front (Sherman, 2013) (Fig. 2).

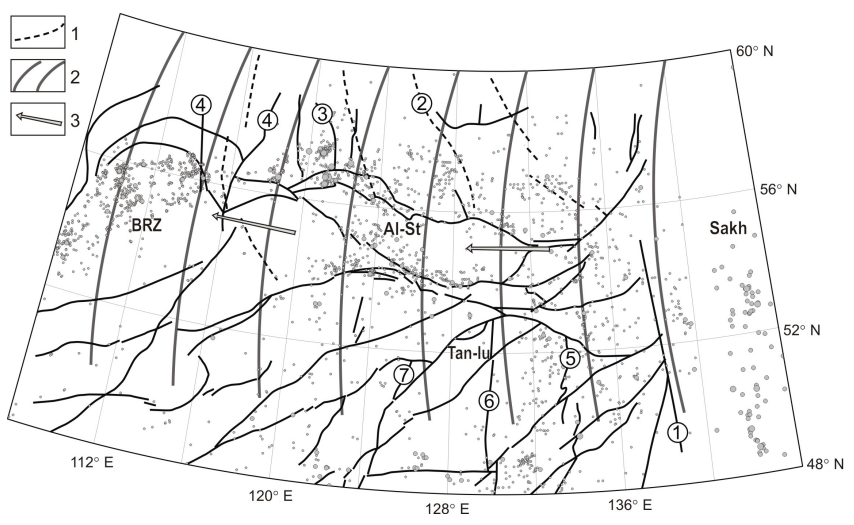

Figure 2. The spatial distribution of seismicity in the annual cycles with respect to the strain wave fronts and meridional structures. Active tectonic faulting: Tanlu fault zone, Aldan-Stanovoy block (Al-St) and Baikal rift zone (BRZ). Figures in the circles denote the faults: 1 - Limurchan, 2 - Tyrkanda, 3 - Temulyakit meridional faults, 4 - meridional structures of the eastern flank of the Baikal rift zone, 5 - Gastakh, 6 - western Turanian, 7 - LevoMinsky. 1 - submeridional interblock faults of the Aldan shield; 2 strain wave fronts (Sherman, 2013); 3 - the direction of seismicity maxima migration in the annual cycles and movements of the strain wave fronts.

The displacement rate values for seismicity maxima are obtained from regression equations using the linear approximation method and are equal to $U_{\mathrm{A}}=-950 \mathrm{~km} \mathrm{yr}^{-1}, U_{\mathrm{B}}=$ $-1170 \mathrm{~km} \mathrm{yr}^{-1}, U_{\mathrm{C}}=-986 \mathrm{~km} \mathrm{yr}^{-1}, U_{\mathrm{D}}=-953 \mathrm{~km} \mathrm{yr}^{-1}$, $U_{\mathrm{E}}=-1033 \mathrm{~km} \mathrm{yr}^{-1}$ and $U_{\mathrm{F}}=-725 \mathrm{~km} \mathrm{yr}^{-1}$ for spatial cycles A, B, C, D, E and F, respectively. The minus sign means the westward displacement of the seismicity maxima.

For the entire northeastern segment, the average value of the velocity modulus of the seismicity maxima displacement (with a relative determination error of $7 \%$ ) is equal to $U_{\mathrm{A}-\mathrm{C}}=1000-1022 \mathrm{~km} \mathrm{yr}^{-1}$, whereas for the northwestern segment this value is $U_{\mathrm{D}-\mathrm{F}} \approx$ $(913 \pm 110) \mathrm{km} \mathrm{yr}^{-1}$. The seismicity maxima displacement rate value is $U_{\mathrm{A}-\mathrm{F}} \approx(979 \pm 124) \mathrm{km} \mathrm{yr}^{-1}$, or about $1000 \mathrm{~km} \mathrm{yr}^{-1}$ along the entire northern boundary of the Amurian plate.

\section{The slow strain wave effects inferred from GPS observations}

To explore the deformation processes in the geological medium with a discrete blocky structure and to perform special GPS experimental observations, we selected the South Yakutia geodynamic polygon located near the northern boundary of the Amurian plate, at the junction of two major tectonic structures - the Aldan Shield and the Stanovoy Range. Recently, a number of blocks of different sizes and configurations have been inferred here from geological data. These blocks experience the vertical and horizontal 
movements of different directions, velocities and amplitudes (Imaeva et al., 2012), which are responsible for the complicated character of tectonic movements.

We have analyzed a set of time series obtained at two collocated GPS sites NRGR and NRG2 situated near the active fault intersection area in the central part of the Stanovoy Range (Fig. 1). The NRGR site is located in the area of the Chulman depression on a $15 \times 20 \mathrm{~km}^{2}$ size microblock and is involved in different types of crustal movements and deformations consistent with the kinematics of the bordering active faults (Trofimenko and Bykov, 2014). Site NRG2's location is approximately $2 \mathrm{~km}$ south of the NRGR site and closer to the zone of influence of the active Berkakit fault. The GPS time series obtained at stations NRGR and NRG2 for the horizontal and vertical components are shown in Fig. 3. The stable long-period displacement component is typical of both observation sites in the southeastern direction. For the vertical and horizontal components observed in other directions, the course of the annual displacements is absolutely different. At the two observation sites, the horizontal displacement components in the north-south direction are represented by in-phase curves that can be approximated by a sinusoid (Fig. 3a), which is consistent with the approximation suggested in Serpelloni et al. (2013). The vertical and horizontal displacement components in the eastwest direction vary in an anti-phase manner during separate periods of measurements (Fig. 3b, c), which contradicts the common dynamics of long-period components. The shapes of these curves for the horizontal displacement components are appreciably different from a sinusoid (Trofimenko et al., 2016b).

It is necessary to emphasize that the meteorological factors in the annual cycles influence the shapes of the movement trajectories of the collocated sites equally (van Dam et al., 1994). Therefore, the detected paradox cannot be explained by the meteorological causes.

This paradox can only be resolved in the case when the observation sites are adjacent to the boundaries of specific "hinge" - type local faults (Fig. 4a). Really, for site NRGR, a local feathering fault of the Sunnangyn-Larba northeasttrending fault system is the "hinge", whereas for site NRG2, the" hinge" is one of the branches of the Berkakit northwesttrending fault (Fig. 1). The physical model of this faultblocky structure can be represented as a set of rods - physical pendulums (Fig. 4b) - whose lower parts are fixed, while the upper parts are disturbed from the equilibrium condition. In this case, the upper parts of the rods (blocks) are displaced with respect to some central line (the fault hinge).

The approximation curve fitting for the vertical component of block displacement has led to one more unexpected result. The shape of the best fit function approximating the experimental curve appeared to coincide with a breather - the solution (2) of the sine-Gordon equation (see below). When selecting the theoretical curve in the shape of a breather (2), this result for the north-south component is obtained at (a)

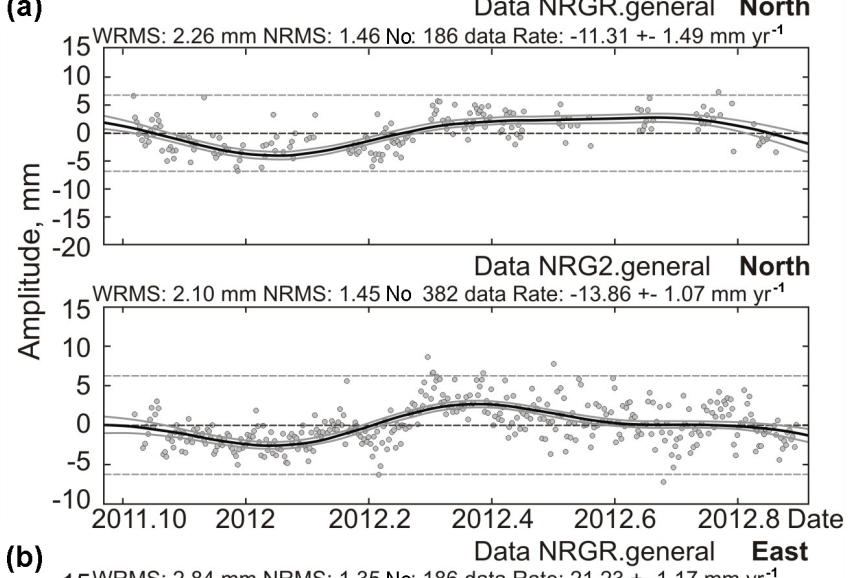

(b)
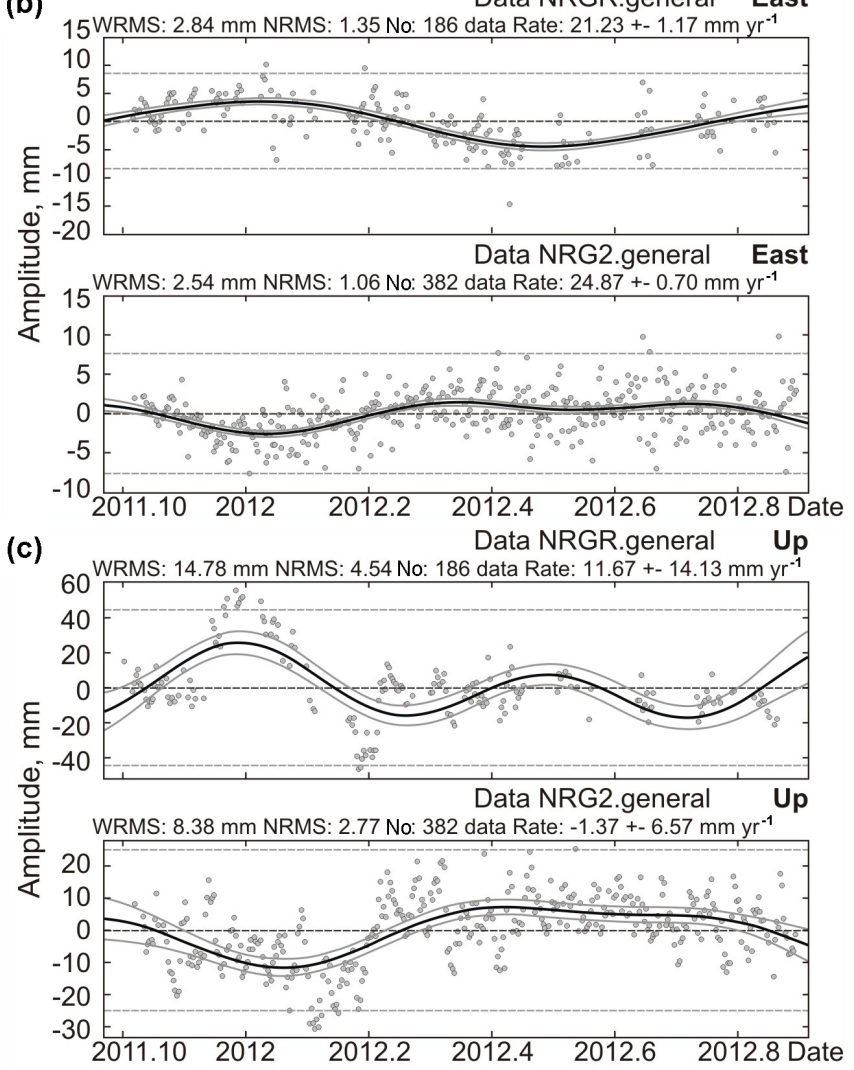

Figure 3. The dynamics of displacement components of NRGR and NRG2 station daily positions in different directions: (a) for the $\mathrm{N}-\mathrm{S}$ components; (b) for the E-W components; (c) for the vertical (updown) components.

$\omega=0.873$ with an error equal to 0.048 (for the sine 0.069 ), and for the east-west component at $\omega=0.780$ with an error equal to 0.052 (for the cosine 0.149). The approximation error of experimental data is calculated from the formula $\sigma=\sqrt{1 / 12 \sum_{k=1}^{12}\left(Y_{k}^{E}-Y_{k}^{T}\right)^{2}}$, where $Y_{k}^{E}-Y_{k}^{T}$ are the residuals between the observed and calculated monthly averaged station positions for the sinusoid and breather. The shapes of 


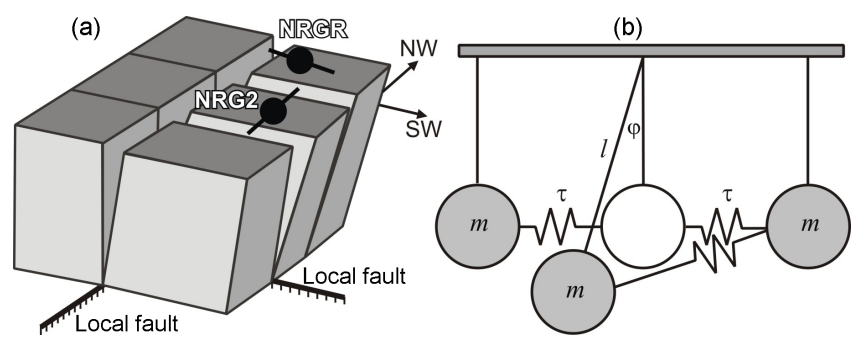

Figure 4. The generalized model of block movement in the vertical plane along differently oriented local faults of the hinge type due to variable vertical loading. (a) The model of block movement along NE- and NW-trending faults and schemes of the full displacement vector decomposition into components. (b) The model of block movement in the shape of coupled pendulums (notations are given in the text).

the fitted curves are shown in Fig. 5; for more details, see Trofimenko et al. (2016b).

The coincidence of the trajectory shape of measured vertical displacements with the shape of a breather, and the correspondence of the blocky structure in the area of GPS site locations to the model of coupled pendulums, served as a motivation for application of the sine-Gordon equation to describe the evolution of the vertical components of block movements.

The mathematical model of quasi-periodical vertical components of oscillations of rigidly coupled crustal blocks with the adjacent "hinge"-type faults corresponds to the equation

$$
\begin{aligned}
\frac{\partial^{2} \phi}{\partial \eta^{2}} & -\frac{\partial^{2} \phi}{\partial \xi^{2}}=\sin \phi, \\
\eta & =\omega t, \xi=x \omega / c, \omega^{2}=m g l / I, c^{2}=\tau d^{2} / I,
\end{aligned}
$$

where $\phi$ is the angle of deviation of the pendulum (rod) from the equilibrium position; $m g l \sin \phi$ is the moment of the gravity force, $m$ is the lumped mass of the pendulum, $l$ is the length of the rod (the height of the block), $\tau d^{2} \frac{\partial^{2} \phi}{\partial x^{2}}$ is the sum of the moments of the torsion forces exerted by the adjacent blocks, $\tau$ is the constant of the spring torsion (rigidity), $d$ is the increment of the interblock distance (increase or decrease depending on the type of movement), and $I$ is the moment of the block inertia.

One of the solutions of Eq. (1) is called a breather (dynamic soliton) and represents a nonlinear function, which for the case of the soliton with the immobile center of gravity can be written as

$\phi(x, t)=4 \tan ^{-1}\left[\left(\frac{\sqrt{1-\omega^{2}}}{\omega}\right) \frac{\sin (\omega t)}{\cosh \left(x \sqrt{1-\omega^{2}}\right)}\right]$,

where $\omega$ is the inner frequency of the breather, $x$ determines the origin of the curve and $t$ is the independent variable (time).

Like a soliton, the breather has the shape of an impulse; it is localized in space and is pulsating in time. In the low-frequency range $\omega \ll 1$ the breather can be qualitatively treated as a weakly coupled kink-antikink pair (the sineGordon equation solutions of opposite signs in the shape of a topological soliton - a wave with a changeless profile in the shape of a kink) (Braun and Kivshar, 2004).

The detected high correlation of the observed site displacement trajectories with the theoretical curve corresponding to a breather allows us to suggest that the mechanism of these oscillations can be associated with the occurrence of strain waves in the fault intersection system. In this case, these waves can be qualitatively treated as standing waves of compression-extension in the blocky geological medium.

The sine-Gordon equation solution in the shape of a breather has previously been applied for modeling the wave dynamics of faults and strain waves (Mikhailov and Nikolaevskiy, 2000; Gershenzon et al., 2009; Erickson et al., 2011). Mikhailov and Nikolaevskiy (2000) considered a scenario when collision of two tectonic waves (kink-antikink collision) resulted in the occurrence of a large earthquake. The solution in the shape of a breather has also been applied for the interpretation of the features of fault dynamics observed after the 1989 Loma Prieta earthquake (Gershenzon et al., 2009). Based on a modified Burridge-Knopoff model, a solution has been obtained that corresponds to a localized failure - a breather that propagates along a fault and is damping in the fault segment of the final length (Erickson et al., 2011). Wu and Chen (1998) earlier reduced a onedimensional Burridge-Knopoff spring-block model to the sine-Gordon equation and applied its solution in the shape of a solitary wave (kink) to investigate earthquakes.

\section{Concluding remarks}

The accumulated facts indicate the propagation of slow wave-like movements within the crust and the lithosphere at different velocities on global and regional scales (Bykov, 2014). The results of our investigation (the periodicity of the seismic components, spatial cycles with phase shift of seismicity maxima, migration velocity of earthquake epicenters) and their comparison with the known data allow us to identify the dynamics of seismicity along the northern boundary of the Amurian plate as a wave process. We have revealed synchronous quasi-periodic seismicity variations in equally spaced clusters with spatial periods of 7.26 and $3.5^{\circ}$ that are comparable with the length of slow strain waves $(\lambda=250$ $450 \mathrm{~km})$ detected in the Eurasian and Amurian tectonic plate interaction area $\left(107-140^{\circ} \mathrm{E}\right)$ (Sherman, 2013). The slow strain wave velocity in Pribaikalya and Priamurye attains 5$20 \mathrm{~km} \mathrm{yr}^{-1}$ and is comparable with the migration velocity of crustal deformations $\left(10-100 \mathrm{~km} \mathrm{yr}^{-1}\right)$ from the JapanKuril-Kamchatka subduction zone (Ishii et al., 1978; Kasahara, 1979; Yoshioka et al., 2015).

The calculated average displacement rate value of the maxima of weak seismicity $(2 \leq M \leq 4)$ along the north- 

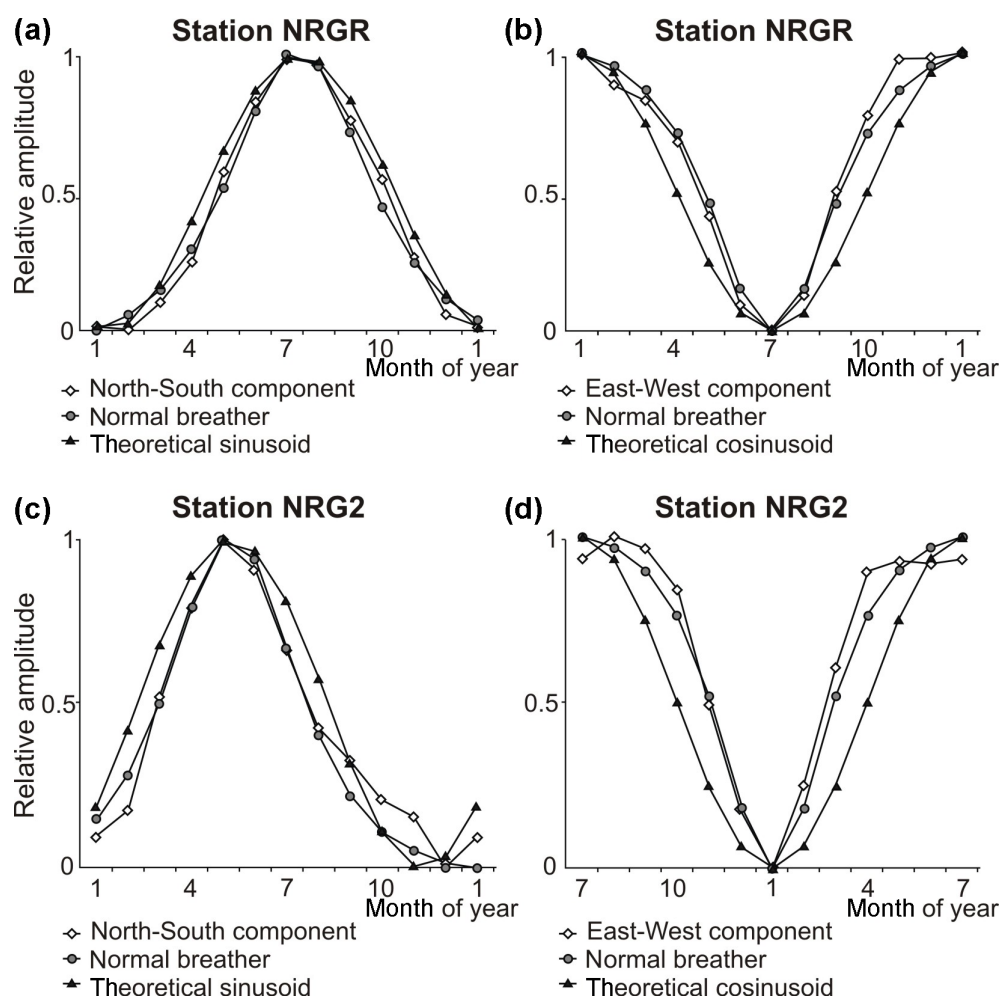

Figure 5. Seasonal variations of NRGR and NRG2 station positions. Approximation of the observed displacement curves by the theoretical curves for the N-S (a) and E-W (b) components at the NRGR site, and for the N-S (c) and E-W (d) components at the NRG2 site.

ern boundary of the Amurian plate is about $1000 \mathrm{~km} \mathrm{yr}^{-1}$, which is 2 orders of magnitude larger than the velocity of slow strain waves $\left(\sim 10-100 \mathrm{~km} \mathrm{yr}^{-1}\right)$. This may imply that slow strain waves modulate variations of weak seismicity $(2 \leq M \leq 4)$ during the year.

The displacement of seismicity in the annual cycles occurs from east to west and coincides with the direction of migration of large earthquakes, strain wave fronts and crustal deformation detected from direct deformographic and GPS measurements (Kasahara, 1979; Bella et al., 1990; Harada et al., 2003; Yoshioka et al., 2015). The slow strain wave fronts are triggers of large earthquakes $(M>6)$ in the submeridional faults of the Amurian plate.

The spatial correlation of migration of seismicity and deformations as well as the migration of deformations - two different manifestations of the geodynamic process - may mean that seismicity migration is associated with the propagation of tectonic stresses in the form of slow strain waves that cause a complementary load and subsequent earthquake occurrence. The numerous results of observations of seismicity migration are hard to explain by causes other than wavelike variations of the global and local stress fields.

The conclusions on the wave pattern of the deformation process are consistent with the results of special experimental observations performed to explore crustal block interaction. The seasonal course of displacements of GPS stations
NRGR and NRG2, involved in the in situ experimental observations, or of the deformations of the blocky structure of the crust, exhibits a wave-like rather than linear pattern. The wave-like displacements can be explained by transmittance of slow strain waves.

Based upon the statistical modeling, we have established the in-phase and anti-phase changes of the components of the full displacement vector, the relative time delay of the maxima and minima for separate components, and dissimilarity of the displacement trajectory from a sinusoid. In order to describe the evolution of oscillations of the interacting blocks, a simple mathematical model is proposed from which the explanation of the observed specific behavior of these blocks follows.

Based on experimental observation data and the developed model of crustal block movement, we have shown that there is one possible interpretation for this fact that the trajectory of GPS station position disturbance is induced by migration of crustal deformation in the form of slow waves.

\section{Data availability}

The basic data were derived from the Earthquakes of Russia catalog (http://eqru.gsras.ru), the catalog compiled by the Baikal Branch of the Geophysical Survey of the Russian 
Academy of Sciences (GS RAS) (http://www.seis-bykl.ru/) and the IRIS catalog (http://www.iris.edu).

Acknowledgements. We are deeply grateful to the editor Arcady Dyskin and to two reviewers, Semen Sherman and the anonymous reviewer, for constructive comments on the first version of the paper. The reported study was funded by the RFBR according to research project no. 16-05-00097 a.

Edited by: A. Dyskin

Reviewed by: S. Sherman and one anonymous referee

\section{References}

Anderson, D. L.: Accelerated plate tectonics, Science, 187, 10771079, 1975.

Barabanov, V. L., Grinevsky, A. O., Kissin, I. G., and Mil'kis, M. P.: Hydrogeological and seismic effects of deformational waves in the foremost Kopet Dag fault zone, Izvestiya Akademii Nauk SSSR, Fizika Zemli, 5, 21-31, 1988.

Bazavluk, T. A. and Yudakhin, F. N.: Deformation waves in Earth crust of Tien Shan on seismological data, Dokl. Akad. Nauk, 329, 565-570, 1993.

Bella, F., Biagi, P. F., Caputo, M., Della Monica, G., Ermini, A., Manjgaladze, P., Sgrigna, V., and Zilpimian, D.: Very slowmoving crustal strain disturbances, Tectonophysics, 179, 131139, 1990.

Bormotov, V. A. and Bykov, V. G.: Seismological monitoring of the deformation process, Geol. Pac. Ocean, 16, 981-994, 2001.

Bott, M. H. P. and Dean, D. S.: Stress diffusion from plate boundaries, Nature, 243, 339-341, 1973.

Braun, O. M. and Kivshar, Y. S.: The Frenkel-Kontorova model: concepts, methods, and applications, Berlin: Springer, 472 pp., 2004.

Bykov, V. G.: Strain waves in the Earth: Theory, field data, and models, Russ. Geol. Geophys., 46, 1158-1170, 2005.

Bykov, V. G.: Stick-slip and strain waves in the physics of earthquake rupture: experiments and models, Acta Geophys., 56, 270285, 2008.

Bykov, V. G.: Sine-Gordon equation and its application to tectonic stress transfer, J. Seismol., 18, 497-510, 2014.

Elsasser, W. M.: Convection and stress propagation in the upper mantle, in: The Application of Modern Physics to the Earth and Planetary Interiors, edited by: Runcorn, S. K., 223-246, WileyInterscience, New York, 1969.

Erickson, B. A., Birnir, B., and Lavallee, D.: Periodicity, chaos and localization in a Burridge-Knopoff model of an earthquake with rate-and-state friction, Geophys. J. Intern., 187, 178-198, 2011.

Gershenzon, N. I., Bykov, V. G., and Bambakidis, G.: Strain waves, earthquakes, slow earthquakes, and afterslip in the framework of the Frenkel-Kontorova model, Phys. Rev. E, 79, 056601, doi:10.1103/PhysRevE.79.056601, 2009.

Gorbunova, E. A. and Sherman, S. I.: Slow deformation waves in the lithosphere: Registration, parameters, and geodynamic analysis (Central Asia), Russ. J. Pac. Geol., 6, 13-20, 2012.

Harada, M., Furuzawa, T., Teraishi, M., and Ohya, F.: Temporal and spatial correlations of the strain field in tectonic active region, southern Kyusyu, Japan, J. Geodyn., 35, 471-481, 2003.
Ida, Y.: Slow-moving deformation pulses along tectonic faults, Phys. Earth Planet. Inter., 9, 328-337, 1974.

Imaeva, L. P., Imaev, V. S., and Koz'min, B. M.: Seismogeodynamics of the Aldan-Stanovoi block, Russ. J. Pac. Geol., 6, 1-12, 2012.

Ishii, H., Sato, T., Tachibana, K., Hashimoto, K., Murakami, E., Mishina, M., Miura, S., Sato, K., and Takagi, A.: Crustal strain, crustal stress and microearthquake activity in the northeastern Japan arc, Tectonophysics, 97, 217-230, 1983.

Kasahara, K.: Migration of crustal deformation, Tectonophysics, 52, 329-341, 1979.

Kissin, I. G.: Hydrological effects of deformation waves in the Earth's crust, Geophys. Res. Lett., 9, 43-52, 2008.

Kuz'min, Y. O.: Deformation autowaves in fault zones, Izv. Phys. Solid Earth, 48, 1-16, 2012.

Lee, W. H. K., Celebi, M., Todorovska, M. I., and Igel, H. (Eds): Special Issue on "Supplement. Rotational seismology and engineering applications”, B. Seismol. Soc. Am., 99, 1486 pp., 2009.

Lukk, A. A. and Nersesov, I. L.: Time-dependent parameters of a seismotectonic process, Izvestiya Akademii Nauk SSSR, Fizika Zemli, 3, 10-27, 1982.

Malamud, A. S. and Nikolaevskii, V. N.: The periodicity of PamirsHindukush earthquakes and the tectonic waves in subducted lithosphere plates, Dokl. Akad. Nauk. SSSR, 269, 1075-1078, 1983.

Malamud, A. S. and Nikolaevsky, V. N.: Cyclicity of seismotectonic events in the marginal region of the Indian lithospheric plate, Dokl. Akad. Nauk. SSSR, 282, 1333-1337, 1985.

Mikhailov, D. N. and Nikolaevskiy, V. N.: Tectonic waves of the rotational type generating seismic signals, Izv. Phys. Solid Earth, 36, 895-902, 2000.

Milyukov, V., Mironov, A., Kravchuk, V., Amoruso, A., and Crescentini, L.: Global deformations of the Eurasian plate and variations of the Earth rotation rate, J. Geodyn., 67, 97-105, 2013.

Miura, S., Ishii, H., and Takagi, A.: Migration of vertical deformations and coupling of island arc plate and subducting plate, in: Slow Deformation and Transmission of Stress in the Earth, edited by: Cohen, S. C. and Vanííek, P., 125-138, Washington, D.C. Geoph. Monogr. Series, 49, 140 pp., 1989.

Nevskii, M. V., Morozova, L. A., and Zhurba, M. N.: The effect of propagation of the long-period strain perturbations, Dokl. Akad. Nauk SSSR, 296, 1090-1094, 1987.

Nersesov, I. L., Lukk, A. A., Zhuravlev, V. I., and Galaganov, O. N.: On propagation of strain waves in the crust of southern Central Asia, Izvestiya Akademii Nauk SSSR, Fizika Zemli, 5, 102-112, 1990.

Nikolaevskiy, V. N.: Geomechanics and fluidodynamics, Kluwer, Dordrecht, 349 pp., 1996.

Nikolaevskiy, V. N.: Tectonic stress migration as nonlinear wave process along earth crust faults, in: Proc. of 4th Inter. Workshop on Localization and Bifurcation Theory for Soils and Rocks, Gifu, Japan, 28 September-2 October 1997, edited by: Adachi, T., Oka, F., and Yashima, A., 137-142, Balkema, Rotterdam, 1998.

Press, F. and Allen, C.: Patterns of seismic release in the southern California region, J. Geophys. Res., 100, 6421-6430, 1995.

Reuveni, Y., Kedar, S., Moore, A., and Webb, F.: Analyzing slip events along the Cascadia margin using an improved subdaily 
GPS analysis strategy, Geophys. J. Intern., 198, 1269-1278, 2014.

Scholz, C. H.: A physical interpretation of the Haicheng earthquake prediction, Nature, 267, 121-124, 1977.

Serpelloni, E., Faccenna, C., Spada, G., Dong, D., and Williams, S. D. P.: Vertical GPS ground motion rates in the EuroMediterranean region: New evidence of velocity gradients at different spatial scales along the Nubia-Eurasia plate boundary, J. Geophys. Res., 118, 6003-6024, 2013.

Sherman, S. I.: Deformation waves as a trigger mechanism of seismic activity in seismic zones of the continental lithosphere, Geodynamics \& Tectonophysics, 4, 83-117, 2013.

Sherman, S. I., Sorokin, A. P., Sorokina, A. T., Gorbunova, E. A., and Bormotov, V. A.: New data on the active faults and zones of modern lithosphere destruction in the Amur region, Dokl. Earth Sci., 439, 1146-1151, 2011.

Stein, R. S., Barka, A. A., and Dieterich, J. H.: Progressive failure on the North Anatolian fault since 1939 by earthquake stress triggering, Geophys. J. Intern., 128, 594-604, 1997.

Teisseyre, R., Takeo, M., and Majewski, E. (Eds.): Earthquake source asymmetry, structural media and rotation effects, Springer-Verlag, Berlin, 582 pp., 2006.

Trofimenko, S. V.: Tectonic interpretation of the statistical model of distributions of anomalies azimuths of gravity and magnetic fields of the Aldanian Shield, Geol. Pac. Ocean, 29, 64-77, 2010.

Trofimenko, S. V. and Bykov, V. G.: The model of crustal block movement in the South Yakutia geodynamic testing area based on GPS data, Russ. J. Pac. Geol., 8, 247-255, 2014.
Trofimenko, S. V., Bykov, V. G., and Merkulova, T. V.: Seismicity Migration in the Zone of Convergent Interaction between the Amur Plate and the Eurasian Plate, J. Volcanol. Seismol., 9, 210 222, 2015.

Trofimenko, S. V., Bykov, V. G., and Merkulova, T. V.: Spacetime model for migration of weak earthquakes along the northern boundary of the Amurian microplate, J. Seismol. doi:10.1007/s10950-016-9600-x, 2016a.

Trofimenko, S. V., Bykov, V. G., Shestakov, N. V., Grib, N. N., and Takahashi, H.: A new insight into the nature of seasonal variations in coordinate time series of GPS sites located near active faults, Front. Earth Sci., 10, 560-569, 2016b.

van Dam, T. M., Blewitt, G., and Heflin, M. B.: Atmospheric pressure loading effects on global positioning system coordinate determinations, J. Geophys. Res., 99, 23939-23950, 1994.

Wang, S. and Zhang, Z.: Plastic-flow waves ("slow-waves") and seismic activity in Central-Eastern Asia, Earthquake Res. China, 19, 74-85, 2005.

Wu, Z. L. and Chen, Y. T.: Solitary wave in a Burridge-Knopoff model with slip-dependent friction as a clue to understanding the mechanism of the self-healing slip pulse in an earthquake rupture process, Nonlin. Processes Geophys., 5, 121-125, doi:10.5194/npg-5-121-1998, 1998.

Yoshioka, S., Matsuoka, Y., and Ide, S.: Spatiotemporal slip distributions of three long-term slow slip events beneath the Bungo Channel, southwest Japan, inferred from inversion analyses of GPS data, Geophys. J. Intern., 201, 1437-1455, 2015. 